\title{
Solar wind dependence of ion parameters in the Earth's magnetospheric region calculated from CLUSTER observations
}

\author{
M. H. Denton ${ }^{1,2}$ and M. G. G. T. Taylor ${ }^{2}$ \\ ${ }^{1}$ Department of Communication Systems, Lancaster University, Lancaster, LA1 4WA, UK \\ ${ }^{2}$ Science Operations Department, European Space Agency, 2201AZ Noordwijk ZH, The Netherlands \\ Received: 21 January 2008 - Revised: 4 March 2008 - Accepted: 5 March 2008 - Published: 26 March 2008
}

\begin{abstract}
Moments calculated from the ion distributions $(\sim 0-40 \mathrm{keV})$ measured by the Cluster Ion Spectrometry (CIS) instrument are combined with data from the Cluster Flux Gate Magnetometer (FGM) instrument and used to characterise the bulk properties of the plasma in the nearEarth magnetosphere over five years (2001-2005). Results are presented in the form of 2-D xy, xz and yz GSM cuts through the magnetosphere using data obtained from the Cluster Science Data System (CSDS) and the Cluster Active Archive (CAA). Analysis reveals the distribution of $\sim 0$ $40 \mathrm{keV}$ ions in the inner magnetosphere is highly ordered and highly responsive to changes in solar wind velocity. Specifically, elevations in temperature are found to occur across the entire nightside plasma sheet region during times of fast solar wind. We demonstrate that the nightside plasma sheet ion temperature at a downtail distance of $\sim 12$ to 19 Earth radii increases by a factor of $\sim 2$ during periods of fast solar wind $\left(500-1000 \mathrm{~km} \mathrm{~s}^{-1}\right)$ compared to periods of slow solar wind $\left(100-400 \mathrm{~km} \mathrm{~s}^{-1}\right)$. The spatial extent of these increases are shown in the $x y, x z$ and $y z$ GSM planes. The results from the study have implications for modelling studies and simulations of solar-wind/magnetosphere coupling, which ultimately rely on in situ observations of the plasma sheet properties for input/boundary conditions.
\end{abstract}

Keywords. Magnetospheric physics (Plasma convection; Plasma sheet; General or miscellaneous)

\section{Introduction}

The interaction of the solar wind with the Earth's magnetosphere is highly complex and the manner in which the transport of energy and momentum is facilitated is a primary focus of space physics research. The investigation of these pro-

Correspondence to: M. H. Denton

(m.denton@lancaster.ac.uk) cesses is multi-faceted, involving theory, simulation, groundbased and in-situ observations. These tools are used separately or in combination to reveal the physics acting in the near-Earth environment. In this tradition, the aim of the fourspacecraft Cluster mission (Escoubet et al., 2001) is to examine local three-dimensional, time-varying phenomena in the Earth's magnetosphere and nearby environment (e.g. solar wind, magnetopause, polar cusps, plasma sheet, lobes etc.). Since February 2006, Cluster data have been made publicly available via the Cluster Active Archive or CAA (Perry et al., 2005) and it is this dataset which is utilised in the current study. The initial motivation for the work presented in this paper is to utilise the entire dataset of Cluster measurements via the CAA, and hence to investigate the population of $\sim 0-40 \mathrm{keV}$ ions in the inner magnetosphere.

Measurements of bulk parameters such as $\mathrm{H}^{+}$temperature and density from the on-going Cluster mission are presented in the form of 2-D snapshots averaged over five years (20012005). Extensive statistical studies of the plasma within the near-Earth magnetosphere have previously revealed the behaviour of such parameters (e.g. Baumjohann et al., 1988; Newell and Meng, 1992; Borovsky et al., 1998; Wing and Newell, 1998; Měrka et al., 2002; Tysganenko and Mukai, 2003; Wing et al., 2005; Neagu et al., 2005; Denton et al., 2005, 2006; Borovsky and Denton, 2006a, b; Lavraud et al., 2005, 2006; Thomsen et al., 2007; Nagata et al., 2007; Åsnes et al., 2008) under a variety of conditions. The primary aim of the work outlined in the current paper is to reveal the averaged bulk parameters in the magnetosphere as a first step to a determination of how such parameters evolve during geomagnetic disturbances. Such analyses are particularly aimed at modellers of the Earth's ring current and radiation belts who utilise values of ion temperature, density, and composition from the nightside region as boundary conditions for simulations (e.g. Liemohn et al., 2006; Fok et al., 1999; Jordanova et al., 1997). In particular, this current study is the first step in adding the database of Cluster measurements

Published by Copernicus Publications on behalf of the European Geosciences Union. 
to observations from $\sim 6.6 R_{E}$ (Los Alamos satellites) and $\sim 9 R_{E}$ (Double Star 1) to generate a full, statistical parameterisation of the magnetosphere out to $\sim 20 R_{E}$, primarily on the nightside, under a variety of prevailing solar wind conditions. Improved input to physics models from such statistics should help increase accuracy and predictions, along with providing a better understanding of the physical processes operating within the region.

\section{Data and analysis}

The Cluster Ion Spectrometry (CIS) instrument package (Rème et al., 2001) is installed on all four Cluster spacecraft and comprises the Composition and Distribution Function (CODIF) sensor and the Hot Ion Analyser (HIA) . Measurements of the ion distribution function in the energy range $\sim 0-40 \mathrm{keV}$ allow calculation of moments of the distribution and thus provide averaged values for temperature, density and velocity. These data, in combination with data from the Fluxgate Magnetometer (FGM) instrument package (Balogh et al., 2001) provide derived values such as plasma beta. The data used in the current study were obtained from the Cluster Science Data System (CSDS), the Cluster Active Archive and the high resolution (1-min) OMNI database.

To permit efficient analysis of such an extensive dataset, individual parameters (e.g. temperature/density etc.) from the Cluster instruments are interpolated to a time resolution of 1-min and combined with values from the OMNI database. In this way, a file is produced for each Cluster spacecraft for each year from 2001-2005 (inclusive), with each file containing 59 parameters either measured by Cluster instruments, or pertaining to the geophysical conditions in the magnetosphere (e.g. position, magnetic field, density, temperature, composition, velocity, solar wind speed, solar wind density etc.). Due to the very nature of the measurement technique utilised by the CIS instrument (e.g. a limited energy range), it only measures a partial moment of the full ion distribution. Depending upon ambient plasma conditions, this can affect how well the distributions (and hence the derived moments) represent the actual local plasma conditions. The effect is most obvious when the plasma contains a very hot (or very cold) component with energy beyond the range of the instrument. In such cases one can expect the measured ion densities to underestimate the actual values. These issues should be borne in mind not only when interpreting the results presented below, but when utilising any thermal plasma measurement.

\section{Results and discussion}

To provide visualisation of the parameters derived from the Cluster observations, each measured data point (1 time minute resolution) from a single Cluster spacecraft (C1) from 2001 to 2005, is binned according to the location of the spacecraft, onto a grid with pixel size of $1 \times 1 R_{E}$ and averaged values within each bin are calculated. Plots are produced in the GSM-xy, GSM-xz, and GSM-yz planes. For each of the plots, to allow sufficient data for robust analysis each data point is only included if obtained within a perpendicular distance $\pm 5 R_{e}$ from the plane in question, in a similar manner as that utilised by Escoubet et al. (1997) in their analysis of ISEE-1 data. For example, for plots in the xy-plane, data are included in the binning if the zcoordinate is $>-5 R_{E}$ and $<5 R_{E}$. Following these criteria two-dimensional plots of the required parameters are produced. A selection of results from the entire analysis are presented below. Pixels coloured grey indicate less than 5 data points contribute to the average within the corresponding bin.

Figure 1 shows the averaged (2001-2005) bulk values of (a) $\mathrm{H}^{+}$density, (b) $\mathrm{O}^{+}$density, (c) $\mathrm{He}^{+}$density, (d) $\mathrm{H}^{+}$temperature and the derived values of (e) ion pressure and (f) total magnetic field, measured by the CODIF/FGM sensors on $\mathrm{C} 1$ in the GSM-xy plane. As is already known, the ion density is well-ordered within the magnetosphere. In the energy range $\sim 0-40 \mathrm{keV}$, high densities $\left(\gg 2 \mathrm{~cm}^{-3}\right)$ are evident on the dayside and presumably correspond to regions within the magnetosheath/boundary layer. Low densities $\left(\sim 0.2 \mathrm{~cm}^{-3}\right)$ of $\mathrm{O}^{+}$and $\mathrm{He}^{+}$ions are evident on the dayside and these are likely signatures of material of ionospheric origin. Indeed, higher densities of these minor constituents are measured closer to the Earth although the high density ring of $\mathrm{O}^{+}$at $\sim L=4$ is caused by contamination of this channel by high energy particles from the Earth's radiation belts (I. Dandouras, private communication). On the nightside, the ion density is much lower overall and most of this material is within the plasma sheet. Previous work indicates the overall plasma sheet number density is between 0.4 and $2.0 \mathrm{~cm}^{-3}$ with an average number density $\sim 0.7 \mathrm{~cm}^{-3}$ (Borovsky et al., 1997). In contrast with the density, the nightside temperature is clearly elevated compared to the dayside and, since pressure is calculated here as the product of density and temperature, this results in a roughly smooth distribution of pressure from dayside (left) to nightside (right). The plot of the averaged total magnetic field clearly indicates the influence of the solar wind on the otherwise dipolar nature of the Earth's magnetic field. (A logical future step in the analysis would be to further constrain the binning conditions and compare in situ observations with current magnetic field models under a variety of conditions).

The data presented in Fig. 1 are useful for identifying gross boundaries within the magnetosphere, and the average values of the bulk parameters. However, the magnetosphere is a dynamic entity with different regions and populations constantly evolving both spatially and temporally. Hence, to calculate averaged parameters within specific regions (e.g. plasma sheet, lobes, cusp, neutral sheet) further selection of the data is required. In a recent study of energetic electrons in the plasma sheet, Åsnes et al. (2008) defined identification criteria for such regions based on in-situ values 

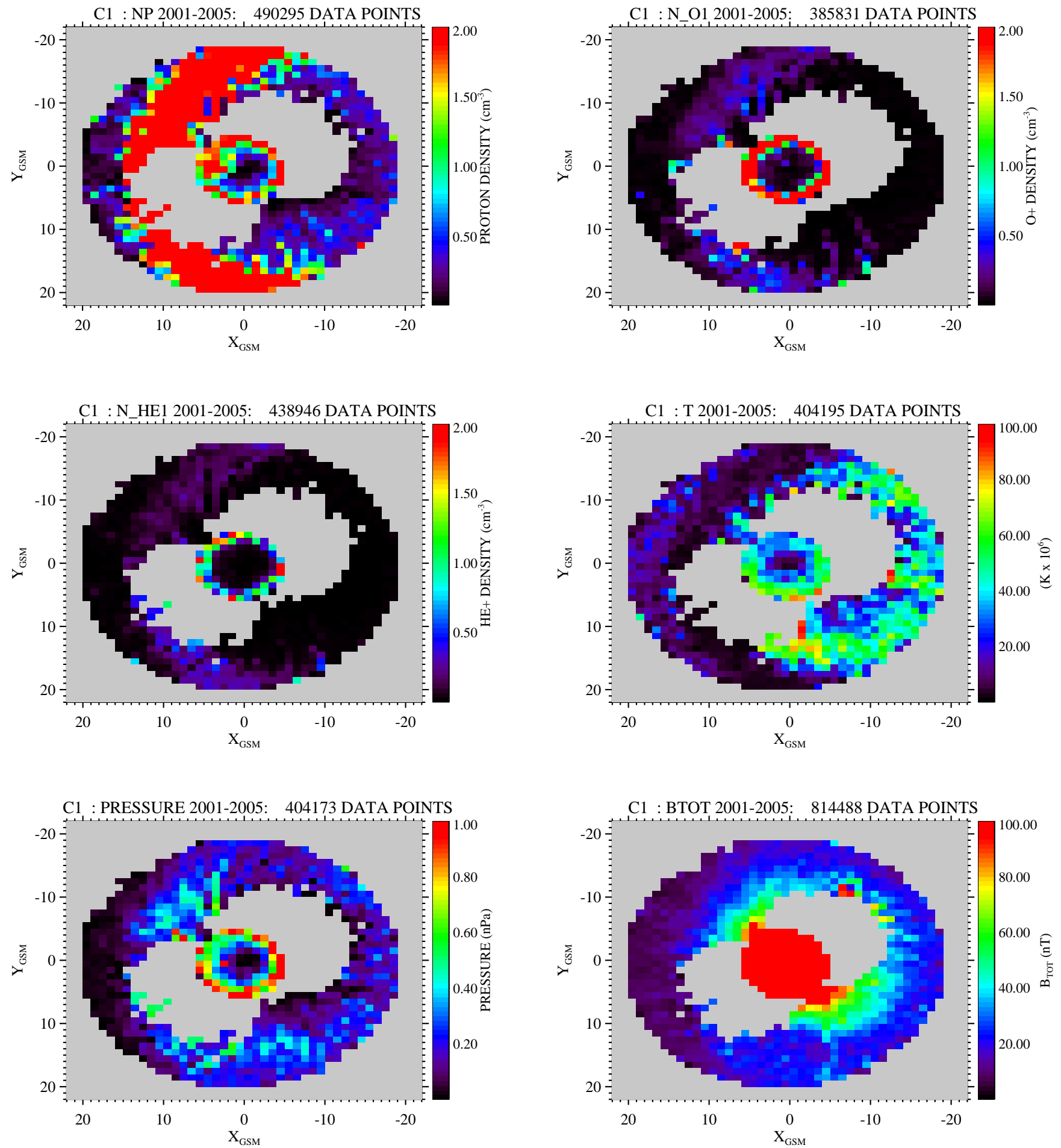

Fig. 1. Averaged (2001-2005) bulk values of (a) $\mathrm{H}^{+}$density, (b) $\mathrm{O}^{+}$density, (c) $\mathrm{He}^{+}$density, (d) $\mathrm{H}^{+}$temperature and the values of (e) ion pressure and (f) total magnetic field strength measured by the CODIF/FGM sensors on C1 in the GSM-xy plane.

of ion parameters such as plasma $\beta$ and ion pressure, $P_{i}$. Figure 2 shows the averaged (2001-2005) $\mathrm{H}^{+}$temperature and pressure measured by the CODIF sensor on $\mathrm{C} 1$ in the GSM-xy, GSM-yz and GSM-xz planes. Data are included in the plot if they fulfil "central plasma sheet" identification criteria (plasma $\beta>0.75$ and $P_{i}>0.02 \mathrm{nPa}$ (Åsnes et al., 2008). Clearly, with such criteria implemented, the number of data points contributing to the averages falls significantly. 

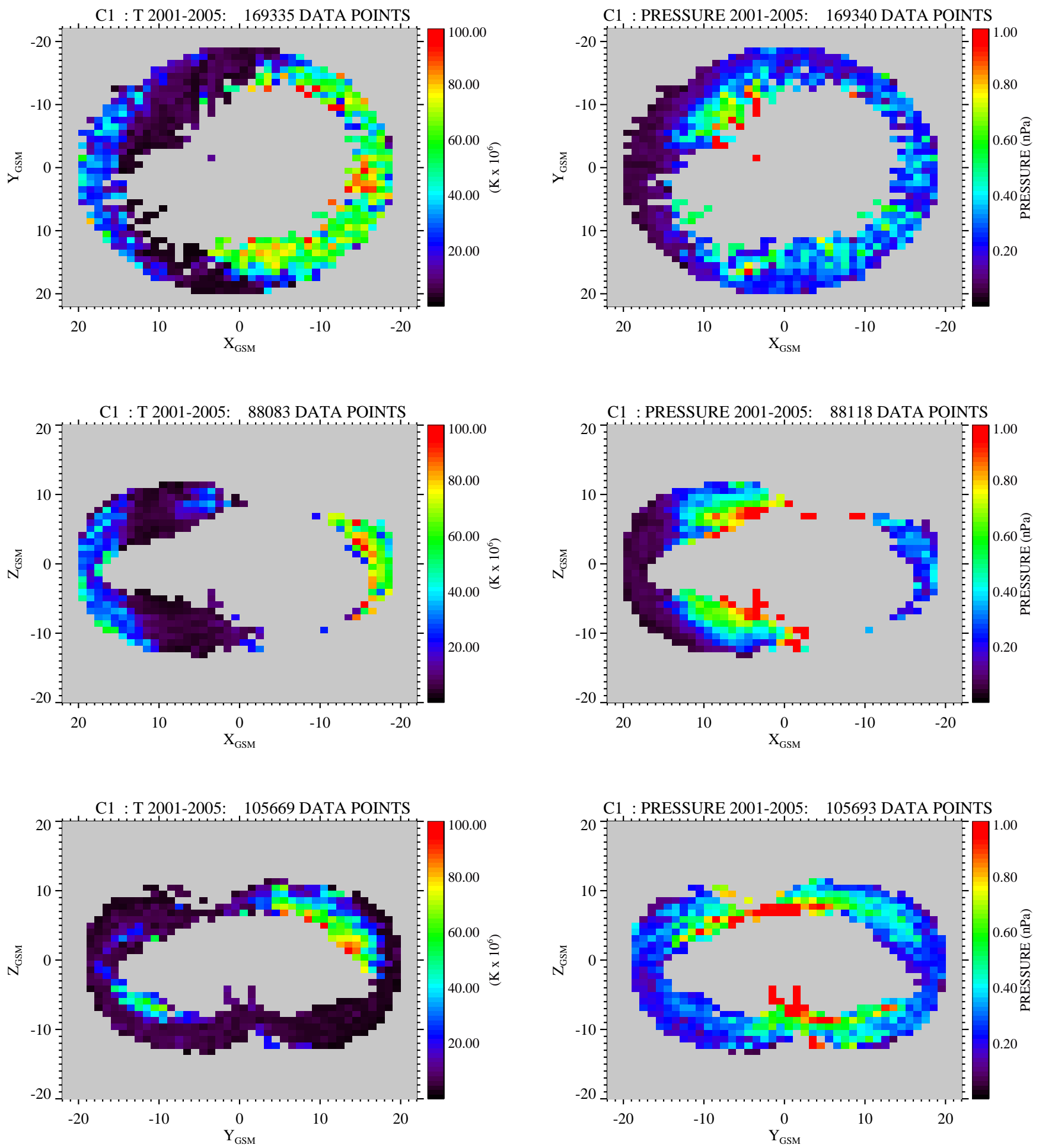

Fig. 2. Averaged plasma sheet $\mathrm{H}^{+}$temperature and pressure in the GSM-xy, GSM-xz and GSM-yz planes averaged from CIS Cluster 1 measurements from 2001-2005. Data are included in the plot if they fulfil central plasma sheet identification criteria (plasma $\beta>0.75$ and $P_{i}>0.02 \mathrm{nPa}(\AA ̊ s n e s$ et al., 2008). 

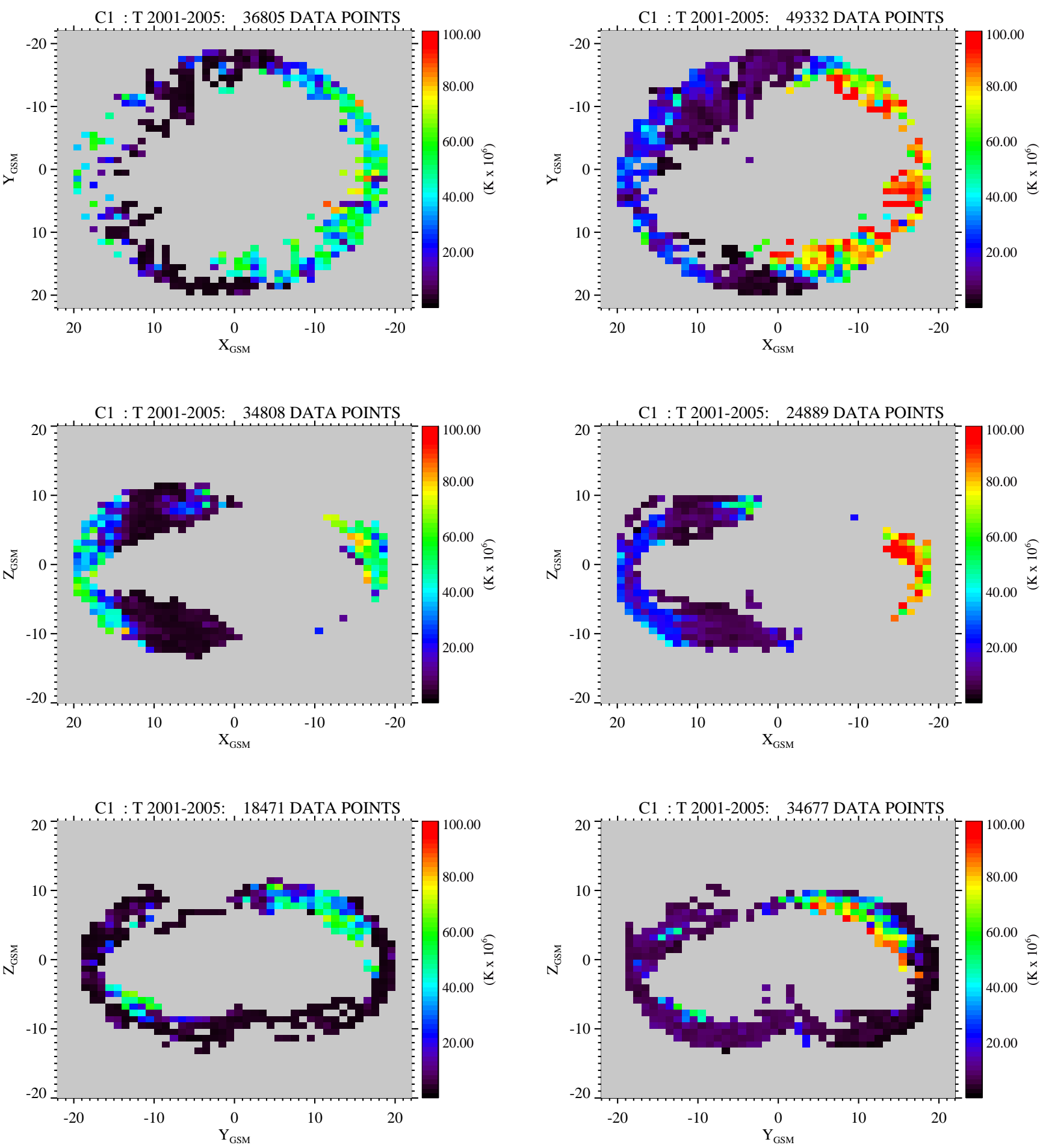

Fig. 3. Averaged $\mathrm{H}^{+}$temperature in the GSM-xy, GSM-xz and GSM-yz planes averaged from CIS Cluster 1 measurements from 2001-2005 for slow (left) and fast (right) solar wind speeds. Data are included in the plot if they fulfil central plasma sheet identification criteria (plasma $\beta>0.75$ and $P_{i}>0.02 \mathrm{nPa}$ ( $\AA$ snes et al., 2008). Clearly, plasma sheet temperature is elevated significantly during fast solar wind.

However, it remains clear that the central plasma sheet, identified using these criteria, is regularly sampled between $\pm 5 R_{E}$ in the $z=0$ GSM-plane.
Figure 3 shows a comparison between the averaged (20012005) $\mathrm{H}^{+}$temperature and ion pressure in the plasma sheet measured by the CODIF sensor on C1 in the GSM-xy, 
GSM-yz and GSM-xz planes. Data are included in the plot if they fulfil central plasma sheet identification criteria (plasma $\beta>0.75$ and $P_{i}>0.02 \mathrm{nPa}$ (see Table 1 in Åsnes et al., 2008). These criteria are implemented to separate central plasma sheet intervals from other regions within the magnetosheath (e.g. outer plasma sheet, lobe, neutral sheet etc.). In addition, each data point is assigned a value of lagged solar wind speed (from the OMNI2 1-minute resolution dataset). Periods of "slow" solar wind (left column) are defined as $100<V_{s w}$ $<400 \mathrm{~km} \mathrm{~s}^{-1}$. Periods of "fast" solar wind (right column) are defined as $500<V_{s w}<1000 \mathrm{~km} \mathrm{~s}^{-1}$. As previously reported by Borovsky et al. (1998) using a limited set of Los Alamos ISEE satellite observations close to local midnight, the ram energy of the solar wind is strongly correlated with the temperature of the magnetosheath, and the measured temperature in the near and distant plasma sheet. Plasma, largely of solar wind origin, is captured during dayside reconnection events, and subsequently transported to the nightside plasma sheet. Statistical work by Tsyganenko and Mukai (2003) quantified this finding using fits to data from the Geotail satellite. The plots in Fig. 3 expand upon previous results by demonstrating that the average ion temperature across the entire nightside plasma sheet is approximatly doubled during periods of fast solar wind compared to periods during slow solar wind. In addition, the results in Fig. 3 demonstrate the spatial extent of the elevated plasma sheet temperature during fast solar wind in the GSM-xy, GSM-yz and GSM$\mathrm{xz}$ planes. This result has implications for any subsequent storm-intervals which occur when this hot plasma sheet material is transported into the inner magnetosphere. As a result of gradient-curvature-drift effects the extra-hot ions are prevented from convecting deep into the dipole where their pressure can be amplified (e.g. Ebihara and Ejiri, 2000; Liemohn et al., 2001; Kozyra and Liemohn, 2003). This likely contributes to the fact that high-speed-stream-driven storms (typically storms following coronal interaction regions in the solar wind), with extended periods of fast solar wind and an enhanced hot ion plasma sheet, tend to have poor $D_{s t}$ signatures (e.g. Borovsky and Denton, 2006; McPherron and Weygand, 2006). Simulated energetic neutral atom (ENA) fluxes indicate the morphology of the plasma sheet is changed little when the plasma sheet temperature is raised - rather the high-energy tail of the ion distribution is raised, resulting in the peak ENA flux originating from roughly the same MLT as when the plasma sheet temperature is low (Ebihara and Fok, 2004).

\section{Discussion and summary}

The initial motivation for the work presented in this paper is to utilise the entire dataset of Cluster measurements via the CAA, and hence to investigate the population of $\sim 0-40 \mathrm{keV}$ ions in the inner magnetosphere. In doing so we have further revealed an important feature of the nightside plasma sheet and its response to upstream solar wind conditions. Extended periods of fast solar wind occur primarily during high speed solar wind streams (e.g. Tsurutani et al., 2006; Denton et al., 2008) during which magnetospheric phenomena such as radiation belt energisation, prolonged magnetospheric convection and enhanced particle precipitation also occur. The nightside plasma sheet is the source population for the ring current, and thus it is important to characterise this population which subsequently is convected into the inner magnetosphere. The ability to differentiate the species which makes up this source population is crucial in obtaining a fuller understanding and prediction of the storm-time response of the inner magnetosphere. As such, the CAA represents an ideal resource with which to determine the effect a heavy-ion-rich plasma (high $\mathrm{O}+$ content) may have upon inner magnetosphere properties and evolution - particularly the dynamics of the ring current.

Clearly, with such an abundance of data, only a subset of results can be presented, and numerous avenues are open for future study. Recent work using the database of Los Alamos satellite observations has indicated that a super-hot and super-dense plasma sheet is detected in the initial phase of high speed stream driven storms (Denton and Borovsky, 2008a, $\mathrm{b}^{1}$ ), and that such periods of prolonged fast solar wind drive changes in the cold plasma populations in the inner magnetosphere (Borovsky and Denton, 2008 ${ }^{2}$ ). Future work using the CAA will investigate if such phenomena are also detected by Cluster in the more-distant magnetotail. Although more sophisticated statistical analysis techniques are being investigated, the simple averages described in this paper allow extensive scientific exploitation of the CAA in the first instance. Initially, the statistical analyses of CIS and FGM measurements have revealed the averaged bulk parameters of density, temperature and pressure around the Cluster orbit. More specifically, it has been shown that the temperature in the nightside plasma sheet can almost double during driving of the magnetosphere by fast solar wind compared to driving by slow solar wind. Plasma in the energy range studied in this report $(\sim 0-40 \mathrm{keV})$ is thought to originate from (a) solar wind plasma which has entered the system during solar-wind/magnetosphere coupling events and (b) ionospheric material which has undergone outflow from the ionosphere. Results in the current paper have particular consequences for the density and temperature of the ion plasma sheet, and hence the source populations for regions such as the ring current and radiation belts. Since various magnetospheric models use the bulk plasma properties within the magnetosphere as model input, it is hoped that this study will

\footnotetext{
${ }^{1}$ Denton, M. H. and Borovsky, J. E.: The superdense plasma sheet in the magnetosphere during high-speed stream-driven storms: plasma transport and timescales, J. Atmos. Sol.-Terr. Phy., under review, 2008b.

${ }^{2}$ Borovsky, J. E. and Denton, M. H.: A statistical look at plasmaspheric drainage plumes, J. Geophys. Res., under review, 2008.
} 
aid such simulations and also stimulate comparison with insitu data and collaboration.

Current work is focused in three areas:

1. Subsuming data from other missions (e.g. Double Star 1 $\left(\sim 9 R_{E}\right)$, Los Alamos satellites $\left(6.6 R_{E}\right)$ ) into the database, permitting statistical analysis of the ion population in the region of geospace out to $\sim 20 R_{E}$.

2. Investigating the evolution of density/temperature/magnetic field and the related parameters of plasma beta/pressure etc. during periods of intense geomagnetic activity.

3. Determining the effects of a heavy-ion-rich plasma on ring current formation and evolution.

Acknowledgements. The authors would like to thank all members of the Cluster mission, in particular members of the CIS (PIs: H. Rème/I. Dandouras) and FGM (PIs: A. Balogh/E. Lucek) instrument teams, the Cluster Active Archive and the Cluster Science Data System. We acknowledge the World Data Centre-C1 at RAL, UK and members of the Space Physics Data Facility for the solar wind and geomagnetic indices used in the data analysis. M. H. Denton wishes to thank the ESTEC Faculty for their hospitality during his visit in June 2007 under the Visiting Scientist Programme. The authors wish to thank P. Escoubet (ESTEC) and members of ISR1, Los Alamos National Laboratory for many helpful discussions and also acknowledge the contributions of P. Lazarou, H. Hill, and $\mathrm{R}$. Vasey to the work presented in this paper.

Topical Editor I. A. Daglis thanks Y. Ebihara for his help in evaluating this paper.

\section{References}

Åsnes, A. R., Friedel, W. H., Lavraud, B., Reeves, G. D., Taylor, M. G. G. T., and Daly, P.: Statistical properties of tail plasma sheet electrons above $40 \mathrm{keV}$, J. Geophys. Res., 113, A03202, doi:10.1029/2007JA012502, 2008.

Balogh, A., Carr, C. M., Acuña, M. H., Dunlop, M. W., Beek, T. J., Brown, P., Fornacon, K.-H., Georgescu, E., Glassmeier, K.H., Harris, J., Musmann, G., Oddy, T., and Schwingenschuh, K.: The Cluster magnetic field investigation: overview of in-flight performance and initial results, Ann. Geophys., 19, 1207-1217, 2001,

http://www.ann-geophys.net/19/1207/2001/.

Baumjohann, W., Paschmann, G., Sckopke, N., Cattell, C. A., and Carlson, C. W.: Average ion moments in the plasma sheet boundary layer, J. Geophys. Res., 93, 11 570-11 520, 1988.

Borovsky, J. E., Thomsen, M. F., and McComas, D. J.: The superdense plasma sheet: Plasmaspheric origin, solar wind origin, or ionospheric origin?, J. Geophys. Res., 102, 22 089-22 097, 1997.

Borovsky, J. E., Thomsen, M. F., and Elphic, R. C.: The driving of the plasma sheet by the solar wind, J. Geophys. Res., 103(A8), 17 617-17 639, 1998.

Borovsky, J. E. and Denton, M. H.: Effect of plasmaspheric drainage plumes on solar-wind/magnetosphere coupling, Geophys. Res. Lett., 33, L20101, doi:10.1029/2006GL026519, 2006a.
Borovsky, J. E. and Denton, M. H.: Differences between CMEdriven storms and CIR-driven storms, J. Geophys. Res., 111, A07S08, doi:10.1029/2005JA011447, 2006b.

Denton, M. H. and Borovsky, J. E.: Superposed epoch analysis of high-speed-stream effects at geosynchronous orbit: hot plasma, cold plasma and the solar wind, J. Geophys. Res., doi:10.1029/2007JA012998, in press, 2008a.

Denton, M. H., Thomsen, M. F., Korth, H., Lynch, S., Zhang, J. C., and Liemohn, M. W.: Bulk plasma properties at geosynchronous orbit, J. Geophys. Res., 11, A07223, doi:10.1029/2004JA010861, 2005.

Denton, M. H., Borovsky, J. E., Skoug, R. M., Thomsen, M. F., Lavraud, B., Henderson, M. G., McPherron, R. L., Zhang, J. C., and Liemohn, M. W.: Geomagnetic storms driven by ICMEand CIR-dominated solar wind, J. Geophys. Res., 111, A07S07, doi:1029/2005JA011436, 2006.

Denton, M. H., Borovsky, J. E., Horne, R. B., McPherron, R. L., Morley, S. K., and Tsurutani, B. T.: High speed solar wind streams - magnetospheric activity during prolonged driving, EOS Trans., AGU, 89(7), 62-63, 2008.

Ebihara, Y. and Ejiri, M.: Simulation study on fundamental properties of the storm-time ring current, J. Geophys. Res., 105, 15 843-15 859, 2000.

Ebihara, Y. and Fok, M.-C.: Postmidnight storm-time enhancement of tens-of-keV proton flux, J. Geophys. Res., 109, A12209, doi:10.1029/2004JA010523, 2004.

Escoubet, C. P., Fehringer, M., and Goldstein, M.: Introduction: the Cluster mission, Ann. Geophys., 19, 1197-1200, 2001, http://www.ann-geophys.net/19/1197/2001/.

Escoubet, C. P., Pedersen, A., Schmidt, R., and Lindqvist, P. A.: Density in the magnetosphere inferred from ISEE-1 spacecraft potential, J. Geophys. Res., 102(A8), 17 595-17 609, 1997.

Fok, M.-C., Moore, T. E., and Delcourt, D. C.: Modelling of inner plasma sheet and ring current during substorms, J. Geophys. Res., 104, 14 557-14 569, 1999.

Jordanova., V. K., Kozyra, J. U., Nagy, A. F., and Khazanov, G. V.: Kinetic model of ring current-atmosphere interactions, J. Geophys. Res., 102, 14 279-14 291, 1997.

Kozyra, J. U. and Liemohn, M. W.: Ring current energy input and decay, Space Sci. Rev., 109, 105-131, 2003.

Lavraud, B., Denton, M. H., Thomsen, M. F., Borovsky, J. E., and Friedel, R. H. W.: Superposed epoch analysis of cold, dense plasma access to geosynchronous orbit, Ann. Geophys., 23, 2519-2529, 2005, http://www.ann-geophys.net/23/2519/2005/.

Lavraud, B., Thomsen, M. F., Wing, S., Fujimoto, M., Denton, M. H., Borovsky, J. E., Åsnes, A., Seki, K., and Weygand, J. M.: Observations of two distinct cold, dense ion populations at geosynchronous is orbit: local time asymmetry, solar wind dependence and origin, Ann. Geophys, 24, 3451-3465, 2006.

Liemohn, M. W., Kozyra, J. U., Thomsen, M. F., Roeder, J. L., Lu, G., Borovsky, J. E., and Cayton, T. E.: Dominant role of the asymmetric ring current in producing stormtime Dst*, J. Geophys. Res., 106, 10 883-10 904, 2001.

Liemohn, M. W., Ridley, A. J., Kozyra, J. U., Gallagher, D. L., Thomsen, M. F., Henderson, M. G., Denton, M. H., Brandt, P. C., and Goldstein, J.: Analyzing the electric field morphology through data-model comparisons of the Geospace Environment Modeling Inner Magnetosphere/Storm 
Assessment Challenge events, J. Geophys. Res., 111, A11S11, doi:10.1029/2006JA011700, 2006.

McPherron, R. L. and Weygand, J.: The solar wind and geomagnetic activity as a function of time relative to corotating interaction regions, in: Recurrent Magnetic Storms, edited by: Tsurutani, B., McPherron, R., Gonzalez, W., Lu, G., Sobral, J. H. A., and Gopalswamy, N., pg. 125, AGU, 2006.

Měrka, J., Safrankova, J., and Nemecek, Z.: Cusp-like plasma in high altitudes: A statistical study of the width and location of the cusp from MAGION-4, Ann. Geophys., 20, 311-320, 2002, http://www.ann-geophys.net/20/311/2002/.

Nagata, D., Machida, S., Ohtani, S., Saito, Y., and Mukai, T.: Solar wind control of plasma number density in the near-Earth plasma sheet, J. Geophys. Res., 112, A09204, doi:10.1029/2007JA012284, 2007.

Neagu, E., Borovsky, J. E., Thomsen, M. F., Gary, S. P., Baumjohann, W., and Treumann, R. A.: Statistical survey of magnetic field and ion velocity fluctuations in the near-Earth plasma sheet Active Magnetospheric Particle Trace Explorers/Ion Release Module (AMPTE/IRM) measurements, J. Geophys. Res., 107(A7), 1098, doi:10.1029/2001JA000318, 2002.

Newell, P. T. and Meng, C.-I.: Mapping the dayside ionosphere to the magnetosphere according to particle precipitation characteristics, Geophys. Res. Lett., 19, 609-612, 1992.

Perry, C., Eriksson, T., Escoubet, P., Esson, S., Laakso, H., McCaffrey, S., Sanderson, T., Bowen, H.: The ESA Cluster Active Archive, Proceedings of the Cluster and Double Star Symposium 5th Anniversary of Cluster in Space, ESA SP-598, 2005.
Rème, H., Aoustin, C., Bosqued, J. M., et al.: First multispacecraft ion measurements in and near Earth's magnetosphere with identical Cluster ion spectrometry (CIS experiment), Ann. Geophys., 19, 1303-1354, 2001, http://www.ann-geophys.net/19/1303/2001/.

Thomsen, M. F., Denton, M. H., Lavraud, B., and Bodeau, M.: Statistics of plasma fluxes at geosynchronous orbit over more than a full solar cycle, Space Weather, 5, S03004, doi:10.1029/2006SW000257, 2007.

Tsurutani, B. T., Gonzalez, W. D., Gonzalez, A. L. C., Guarnieri, F. L., Gopalswamy, N., Grande, M., Kamide, Y., Kasahara, Y., Lu, G., Mann, I., McPherron, R., Sørass, F., and Vasyliunas, V.: Corotating solar wind streams and recurrent geomagnetic activity: a review, J. Geophys. Res., 111, A07S01, doi:10.1029/2005JA011273, 2006.

Tsyganenko, N. A. and Mukai, T.: Tail plasma sheet models derived from Geotail particle data, J. Geophys. Res., 108(A3), 1136, doi:10.1029/2002JA009707, 2003.

Wing, S. and Newell, P. T.: Quiet time plasma sheet ion pressure contribution to Birkeland currents, J. Geophys. Res., 105, 77937802, 2000.

Wing, S., Johnson, J. R., Newell, P. T., and Meng, C.-I.: Dawndusk asymmetries, ion spectra , and sources in the northward interplanetary magnetic field plasma sheet, J. Geophys. Res., 110, A08205, doi:10.1029/2005JA011086, 2005. 